\title{
Metallicity and Quasar Outflows
}

\author{
Huiyuan Wang ${ }^{1,2}$, Hongyan Zhou ${ }^{1,2,3}$, Weimin Yuan ${ }^{4}$ and Tinggui Wang ${ }^{1,2}$
}

\begin{abstract}
Correlations are investigated of the outflow strength of quasars, as measured by the blueshift and asymmetry index (BAI) of the C IV line (Wang et al. 2011), with intensities and ratios of broad emission lines, based on composite quasar spectra built from the Sloan Digital Sky Survey. We find that most of the line ratios of other ions to C IV prominently increases with BAI. These behaviors can be well understood in the context of increasing metallicity with BAI. The strength of dominant coolant, C IV line, decreases and weak collisionally excited lines increase with gas metallicity as a result of the competition between different line coolants. Using Si IV+O IV]/C IV as an indicator of gas metallicity, we present, for the first time, a strong correlation between the metallicitiy and the outflow strength of quasars over a wide range of 1.7 to 6.9 times solar abundance. Our result implies that the metallicity plays an important role in the formation of quasar outflows, likely via affecting outflow acceleration. This effect may have a profound impact on galaxy evolution via momentum feedback and chemical enrichment.
\end{abstract}

Subject headings: galaxies: abundances — galaxies: nuclei — line: formation — line: profiles — quasars: emission lines — quasars: general

\section{Introduction}

Outflows appear to be a common phenomenon in quasars. On small scale, mass loss due to outflows is an important component in the overall structure of quasars (Crenshaw et al. 2003, and references therein). Recently, outflows have been regarded as one of the main

\footnotetext{
${ }^{1}$ Key Laboratory for Research in Galaxies and Cosmology, University of Science and Technology of China, Chinese Academy of Sciences, Hefei, Anhui 230026, China; whywang@mail.ustc.edu.cn

${ }^{2}$ Department of Astronomy, University of Science and Technology of China, Hefei, Anhui 230026, China

${ }^{3}$ Polar Research Institute of China, Jinqiao Rd. 451, Shanghai, 200136, China

${ }^{4}$ National Astronomical Observatories, Chinese Academy of Sciences, Beijing 100012, China
} 
components in the unification of various subclasses of quasars (Richards et al. 2011). On large scales, outflows are considered to be able to clear out inter-stellar medium (ISM; Silk \& Rees 1998; Fabian 1999; Di Matteo et al. 2005) and enrich the inter-galactic medium (IGM; Friaca \& Terlevich 1998), and consequently affect the star formation in the host galaxies. Quasar outflows are important for our understanding of the co-evolution of galaxies and their central black holes.

There exist, in quasar spectra, prominent features associated with outflows, e.g. broad absorption lines (BALs) and blueshifted broad emission lines (BELs). Studies on the two different features reveal quite similar dependencies of outflow properties on some fundamental quantities. BAL quasars with larger outflow velocity tend to have higher Eddington ratio and weaker intrinsic X-ray relative to UV (Ganguly et al. 2007; Fan et al. 2009). And the fraction of BAL quasars also increases with Eddington ratio (Ganguly et al. 2007; Zhang et al. 2010). The blueshift of CIV BELs is more strongly correlated with the Eddington ratio than the BAL properties (Wang et al. 2011, hereafter Wang11) and also depend on the shape of the ionizing continuum (Leighly \& Moore 2004; Richards et al. 2011). Furthermore, the blueshift is apparently stronger in radio-quiet quasars than in radio-loud ones (Marziani et al. 1996). These observational results provide strong support for the scenario that the outflow is directly related to the accretion process and driven by radiation pressure.

Is the launch of outflows affected by other factors, in particular the environment where quasars reside in? In this letter, we uncover a strong correlation between the outflow strength and the quasar metallicity. This correlation is actually not unexpected considering that high metallicity can increase the acceleration of outflows. One good example with strong outflows and enriched metallicity is the ultraluminous infrared quasar Q1321+058 (Wang et al. 2009). Since metals are produced by star formation process, this correlation strongly suggests that star formation in galaxies can affect the evolution of the central black holes. The connection between galaxies and black holes might be more intimate and complex than previous expectation.

This letter is organized as follows. In Section 2 we show the sample selection and present composite spectra as a function of the blueshift and asymmetry index (BAI) of the C IV line, which has been shown to be a good indicator of the strength of quasar outflows (Wang11). We also measure the line flux from the composites. In Section 3 we present strong correlations of various line flux ratios with BAI. These results provide strong evidence to support that outflow strength increases with the metallicity. Finally, we summarize the results and discuss their implications in Section 4. 


\section{Sample Selection and Composite Spectra}

\subsection{Sample Selection}

We select quasars in the redshift range $1.7<z<4.0$ from the Fifth Data Release of the Sloan Digital Sky Survey (SDSS; Schneider et al. 2007). The redshift range is chosen in such a way that the C IV line falls in the wavelength coverage of the SDSS spectrograph. To ensure reliable measurements of emission line parameters, we select objects with median signal-to-noise ratio $(\mathrm{S} / \mathrm{N}) \geq 7$ per pixel in the C IV (1450-1700A) spectral region. We discard BAL quasars as cataloged by Scaringi et al. (2009), since BALs would significantly modify the CIV line profile. 12844 quasars meet these criteria. The SDSS spectra are transformed to the rest frame using the improved redshifts for SDSS quasars as computed by Hewett \& Wild (2010, hereafter HW10).

To measure the C IV line, we first fit the local continuum with a power-law in two wavelength windows near $1450 \AA$ and $1695 \AA$. After subtracting the continuum, we fit the residual spectrum around CIV with two Gaussians. Since the red wing of C IV is contaminated by

He II, only the spectral region of $1450-1580 \AA$ is considered in fitting the $\mathrm{C}$ IV line. The fitting results for most of the objects are reasonable according to our visual inspection. However, a small fraction of objects cannot be well fitted by our automated procedure. To minimize the number of outliers, we eliminate objects with unacceptable fitting, i.e. $\chi^{2} /$ d.o.f $>1.5$. This restriction leads to a final working sample of 11268 quasars.

Following Wang11, we use the blueshift and asymmetry index to measure the deviation of the C IV line from an unshifted and symmetric profile. BAI is defined as the flux ratio of the blue part to the total profile, where the blue part is the part of C IV line at wavelengths short of $1549.06 \AA$, the rest-frame wavelength of the C IV doublets. The BAI estimation may be affected by the accuracy of the redshifts which we use to transform the observed spectra to the rest frame. To reduce the possible uncertainty of BAI thus introduced, we adopt the redshifts provided by HW10, which were derived by cross correlating observed spectra with a carefully-constructed template. It has been demonstrated by Wang11 that the redshifts of HW10 do not introduce any significant bias in measuring BAI.

\subsection{Composite-Spectra Construction and Emission-Line Measurement}

With increased S/N, composite spectra are useful for studying weak lines and the overall properties of quasars by averaging out object-to-object variations (Vanden Berk et al. 2001, hereafter VB01; Nagao et al. 2006, hereafter N06). Since we are interested in the relative 
intensities of BELs, the composites are constructed using the arithmetic mean method. We first divide the quasar sample into five equally-sized subsamples according to their BAI. For each quasar, we use the HW10 redshift to deredshift the spectrum. The spectrum is then normalized at $1450 \AA$, rebinned into the same wavelength bins. In Figure 1, we show the composite spectra covering the wavelength range from 1000 to $3000 \AA$. In our sample, BAI is weakly correlated with redshift and luminosity, so the number of quasars in each wavelength bin as a function of wavelength is similar for different composites (Figure 2). At least 220 and 940 spectra are combined in the bins of the composites near $1000 \AA$ and $3000 \AA$, respectively. This enables detections of weak features in the composite spectra at high statistical significance. Following VB01 and Laor et al. (1997), we locate and identify most of significant line features in the composites.

As CIV BAI increases, other high ionization lines, such as HeII, N V and O vI, also significantly shift towards shorter wavelength. Especially, the blue wing of these lines are prominently enhanced as BAI increases, suggesting that BAI is a good indicator of outflow strength. While, in our composites, low ionization lines, such as O I, C III] and Mg II, don't exhibit apparent shift with respect to the rest frame. It is consistent with previous findings (Marziani et al. 1996; Richards et al. 2002; Shen et al. 2007). These results support that there is no significant bias in HW10 redshifts and BAI.

We then measure the fluxes of the metal lines (or line complex) listed in Tab. 1 from the composites. Generally, we first fit the continuum with a power-law in two continuum windows. After subtracting the continuum, we fit the residual line spectrum with multiGaussians model. The central wavelengths of the continuum windows and the number of Gaussians for each line are listed in Table 1. Note that we only use the spectral region of

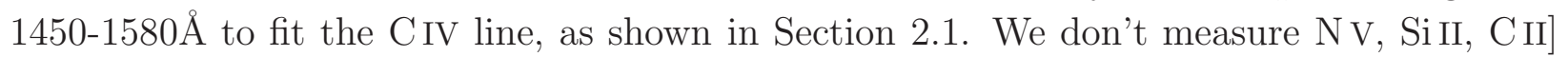
and Mg II (also labelled in Figure 1), because these lines are severely blended with either strong Ly $\alpha$ or strong FeII bump. Due to the uncertainty in the origin of the $1600 \AA$ bump (see N06), it is hard to determine the line profile of HeII. We don't investigate HeII and O III] too.

The errors of the line fluxes shown in Figure 3 are propagated from the uncertainties of the composites. Each composite is built from more than 2000 quasars, the flux errors are therefore very small. The errors include none from the uncertainties in continuum estimate, which is difficult to quantify. However, the continuum placement is important for weak line estimates. In the spectral region of $1690-2030 \AA$, several weak lines are mildly blended. To decompose these lines, we select spectral regions near $1695 \AA$ and $2030 \AA$, where the emission line contribution to the total flux appears to be small, as continuum windows. We mask the region of $1760-1800 \AA$, where Fe II emission is prominent, then simultaneously 
fit the NIV, NIII], Si II+[NeIII] and CIII]+SiIII] lines. Figure 1 shows an example for the spectral decomposition. Our best-fit model reproduces the composite. O I+Si II in small BAI composites appears to lie on the red wing of the strong line complex Ly $\alpha+\mathrm{N} \mathrm{V}$, we therefore adopt local continuums around the line as continuum windows (see Table 1). Nevertheless, the high continuum around O I+Si II at small BAI might be partly contributed by the broad profile of $\mathrm{O} I+\mathrm{Si} I$. If this is the case, the O I+Si II intensity at small BAI is underestimated and more detailed decomposition is required to study its dependence on BAI.

\section{BAI and Metallicity Correlation}

The line fluxes as a function of BAI are shown in the left panel of Figure 3. One can see the line intensities are correlated with BAI, and there seems to exist opposite trends between strong and weak lines. Strong lines (C IV, O VI+Ly $\beta, \mathrm{C}$ III]+Si III] and Si IV+O IV]) either decrease or remain constant with the increase of BAI, while weak lines (the other lines shown in Figure 3) are dramatically enhanced at large BAI. The opposite trends also appears to exist in the lines, which are not measured: the strong line Mg II and the weak lines Si II, Si III] and CII] (see Figure 1 and Wang11). It is well known that line equivalent widths decrease with quasar luminosity, known as the Baldwin effect (Baldwin 1977; Dietrich et al. 2002). If BAI was correlated with luminosity, one would expect that line intensities are correlated with BAI. We compute the median luminosity of each subsample, and find that quasars in the largest-BAI subsample are, on average, only $45 \%$ more luminous than those in the smallest-BAI subsample. Apparently, BAI is weakly related to luminosity 1 and Baldwin effect is not important for the trends reported in this paper.

We then show the line ratios of other ions to CIV as a function of BAI in the right panel of Figure 3. All line ratios, except O VI+Ly $\beta / \mathrm{CIV}$, positively correlate with BAI with a high statistical significance. Among these ratios, Si IV+O IV]/C IV, Al III/C IV, C II/C IV and $\mathrm{CIII}]+\mathrm{SiIII} / \mathrm{C}$ IV have been demonstrated, by the photoionization model, to significantly increase with increasing metallicity (e.g. N06). This means that there exists strong correlation between BAI and metallicity. The intensities of the strong metal lines depend weakly on metallicity because of the thermostat effect introduced by the strong cooling lines

\footnotetext{
${ }^{1}$ N06 found a significant correlation between CIV blueshift and luminosity. We note that they divided the sample into subsamples in a very different way from us. Most of quasars in their sample are assigned into one or two subsamples, while our subsamples are equal size. It might be the most important reason for this difference.
} 
(Korista et al. 1998; Ferland 1999). In metal-rich gas, C IV and O vl2, the most important coolants, even tend to decrease with increasing metallicity because their relative importance as coolants decreases. While, the weak lines can prominently increase with metallicity because the number density of corresponding ions increases and the thermostat effect for them is trivial. Therefore, the metallicity-BAI correlation can well explain the trends of the line intensities shown in the left panel of Figure 3. We note that metallicity can not be the only factor affecting line emission, other factors, such as the ionizing continuum and the overall properties of BELR gas, also play an important role.

Considerable efforts had been devoted to developing line ratios, such as N V/C IV and $\mathrm{SiIV}+\mathrm{OIV}] / \mathrm{CIV}$, as metal abundance diagnostics (Hamann et al. 2002; N06). Here, we adopt $\mathrm{Si}$ IV+O IV]/CIV as a metallicity indicator. Two theoretical line ratio-metallicity relationships, calculated by photoionization models using two types of ionizing continuums, are adopted (N06, Table 10). As shown in Figure 4, the correlation between the inferred metallicity and BAI is weakly dependent on the ionizing continuum. When BAI increases from 0.49 to 0.76 , the metallicity remarkably increases from 1.7 to $6.9 Z_{\odot}$.

We use this indicator rather than that involving $\mathrm{N} \mathrm{V}$ because $\mathrm{Si} \mathrm{IV}+\mathrm{O}$ IV] is a well isolated feature and the origin of the N V line might be complex (Wang et al. 2010a). The theoretical calculation in N06 shows that C II/C IV, AlIII/C IV and C III]+Si III]/C IV are also correlated with metallicity. For comparison, we also show the metallicities inferred from $\mathrm{CIII}]+\mathrm{SiIII} / \mathrm{CIV}$ and $\mathrm{AlIII} / \mathrm{CIV}$ as a function of BAI in Figure 4. The measured ratios of C II to CIV for the two smallest-BAI subsamples are less than the theoretical ratios at $0.2 Z_{\odot}$, which is the lowest metallicity adopted in the theoretical calculation, so we don't calculate the 'C II metalliciy'. One can see that the 'Al III metallicity' is in good agreement with the 'Si IV+O IV] metalliciy', implying that the Al III measurement is reliable. The 'C III]+Si III] metallicity' also increases with BAI, although it is apparently lower than the 'Si IV+O IV] metalliciy'. N06 also found that 'C II and C III]+Si III] metallicities' are much lower than 'AlIII and Si IV+OIV] metallicities'. C II and CIII]+Si III] are emitted by gas with different ionization degree or gas density from C IV. This indicates that CII/C IV and $\mathrm{CIII}]+\mathrm{SiIII}] / \mathrm{CIV}$ are sensitive to the BELR properties adopted by photoionization model, such as the density and spatial distributions of BELR clouds (see N06 for more details). In contrast, Si IV+O IV]/C IV is less sensitive to the variation in the BELR structure, and thus is a more robust metallicity indicator.

\footnotetext{
${ }^{2}$ Note that the measured intensity of $\mathrm{O} V \mathrm{VI}+\mathrm{Ly} \beta$ is severely affected by Ly $\alpha$ forest, its intrinsic intensity should be much higher than the measured value.
} 


\section{Summary and Discussion}

We measure various line fluxes and the blueshift and asymmetry index (BAI) of the C IV line from composite quasar spectra built from the Sloan Digital Sky Survey. Most of investigated line-flux ratios increase significantly with BAI. Based on phototionization models, we interpret these dependencies in terms of stronger outflow in higher gas metallicity environment. This correlation can also account for the positive trends for weak lines and negative trends for strong lines, which are observed in the composites. We then use the flux ratio of $\mathrm{Si}$ IV $+\mathrm{O}$ IV] to $\mathrm{CIV}$ as abundance diagnostics and find that metallicity increases more than 3 times as BAI increases from 0.49 to 0.76 .

We recall that a remarkably similar correlation has been well established in radiationdriven stellar wind (Massey 2003). High metallicity leads to large gas opacity, thus large absorption and acceleration. Recent studies on quasar outflows also favor the paradigm of radiative acceleration (Wang11). So the metallicity-outflow correlation can be naturally understood in terms of the metallicity-acceleration relationship. Another possibility is that starburst in the galactic center can produce abundant metals and drive the gases to fuel the black hole (Wang et al. 2010b). Because outflows are boosted at high accretion rates, the BAI-metallicity relationship can also be produced. The two mechanisms may both work. Although it is difficult to disentangle the predominant physical mechanism from the current data, the first mechanism is apparently more direct. Whichever mechanism dominates, star formation occurred in the host galaxies, which produces metals, has an important impact on the driving of quasar outflow.

The quasar metallicity may dramatically affect the host galaxy properties via feedback. On one hand, outflows from the quasar could exert enough force on the ISM to remove some of the material from the galaxy, consequently inhibiting the growth of the galaxy. This process would be more effective at higher metallicity according to our results. On the other hand, metal-rich outflows and ejected ISM may chemically enrich the IGM as the BELR metallicity is much higher than the solar value (Hamann et al. 2002). The enrichment makes the radiative cooling of the IGM more efficient, and further enhances star formation in galaxies and the growth of their central black holes, in contrast to the momentum feedback process that blows away the ISM. The enrichment rate is proportional to both the mass loss rate of outflows and metallicity, and therefore increases with metallicity in a manner stronger than a linear relationship. This feedback process is particularly important at high redshifts

because 1) the intense star formation at high redshifts may form a substantially metal-rich environment in the galactic nuclei; 2) at high redshifts, IGM is very metal poor and its cooling is less effective. 
We thank the referee for constructive comments that significantly improved the paper. This work is supported by NSFC (11073017, 11033007, 10973013, 10973012), 973 program (2007CB815405, 2009CB824800). Funding for the SDSS and SDSS-II has been provided by the Alfred P. Sloan Foundation, the Participating Institutions, the National Science Foundation, the U.S. Department of Energy, the National Aeronautics and Space Administration, the Japanese Monbukagakusho, the Max Planck Society, and the Higher Education Funding Council for England. The SDSS Web site is http://www.sdss.org/.

\section{REFERENCES}

Baldwin, J. A. 1977, ApJ, 214, 679

Crenshaw, D. M., Kraemer, S. B., \& George, I. M. 2003, ARA\&A, 41, 117

Dietrich, M., Hamann, F., Shields, J. C., et al. 2002, ApJ, 581, 912

Di Matteo, T., Springel, V., \& Hernquist, L. 2005, Nature, 433, 604

Fabian, A. C. 1999, MNRAS, 308, L39

Fan, L. L., Wang, H. Y., Wang, T., Wang, J., Dong, X., Zhang, K., \& Cheng, F. 2009, ApJ, 690, 1006

Ferland, G. 1999, Quasars and Cosmology, 162, 147

Friaca, A. C. S., \& Terlevich, R. J. 1998, MNRAS, 298, 399

Ganguly, R., Brotherton, M. S., Cales, S., et al. 2007, ApJ, 665, 990

Hamann, F., Korista, K. T., Ferland, G. J., Warner, C., \& Baldwin, J. 2002, ApJ, 564, 592

Hewett, P. C., \& Wild, V. 2010, MNRAS, 405, 2302

Korista, K., Baldwin, J., \& Ferland, G. 1998, ApJ, 507, 24

Laor, A., Jannuzi, B. T., Green, R. F., \& Boroson, T. A. 1997, ApJ, 489, 656

Leighly, K. M., \& Moore, J. R. 2004, ApJ, 611, 107

Marziani, P., Sulentic, J. W., Dultzin-Hacyan, D., Calvani, M., \& Moles, M. 1996, ApJS, 104,37

Massey, P. 2003, ARA\&A, 41, 15 
Nagao, T., Marconi, A., \& Maiolino, R. 2006, A\&A, 447, 157

Richards, G. T., Vanden Berk, D. E., Reichard, T. A., Hall, P. B., Schneider, D. P., SubbaRao, M., Thakar, A. R., \& York, D. G. 2002, AJ, 124, 1

Richards, G. T., et al. 2011, AJ, 141, 167

Scaringi, S., Cottis, C. E., Knigge, C., \& Goad, M. R. 2009, MNRAS, 399, 2231

Schneider, D. P., et al. 2007, AJ, 134, 102

Shen, Y., Strauss, M. A., Oguri, M., et al. 2007, AJ, 133, 2222

Silk, J., \& Rees, M. J. 1998, A\&A, 331, L1

Vanden Berk, D. E., Richards, G. T., Bauer, A., et al. 2001, AJ, 122, 549

Wang, H., Wang, T., Yuan, W., et al. 2010a, ApJ, 710, 78

Wang, J.-M., Yan, C.-S., Gao, H.-Q., et al. 2010b, ApJ, 719, L148

Wang, H., Wang, T., Zhou, H., et al. 2011, ApJ, 738, 85

Wang, T., Zhou, H., Yuan, W., Lu, H. L., Dong, X., \& Shan, H. 2009, ApJ, 702, 851

Zhang, S., Wang, T.-G., Wang, H., Zhou, H., Dong, X.-B., \& Wang, J.-G. 2010, ApJ, 714, 367 


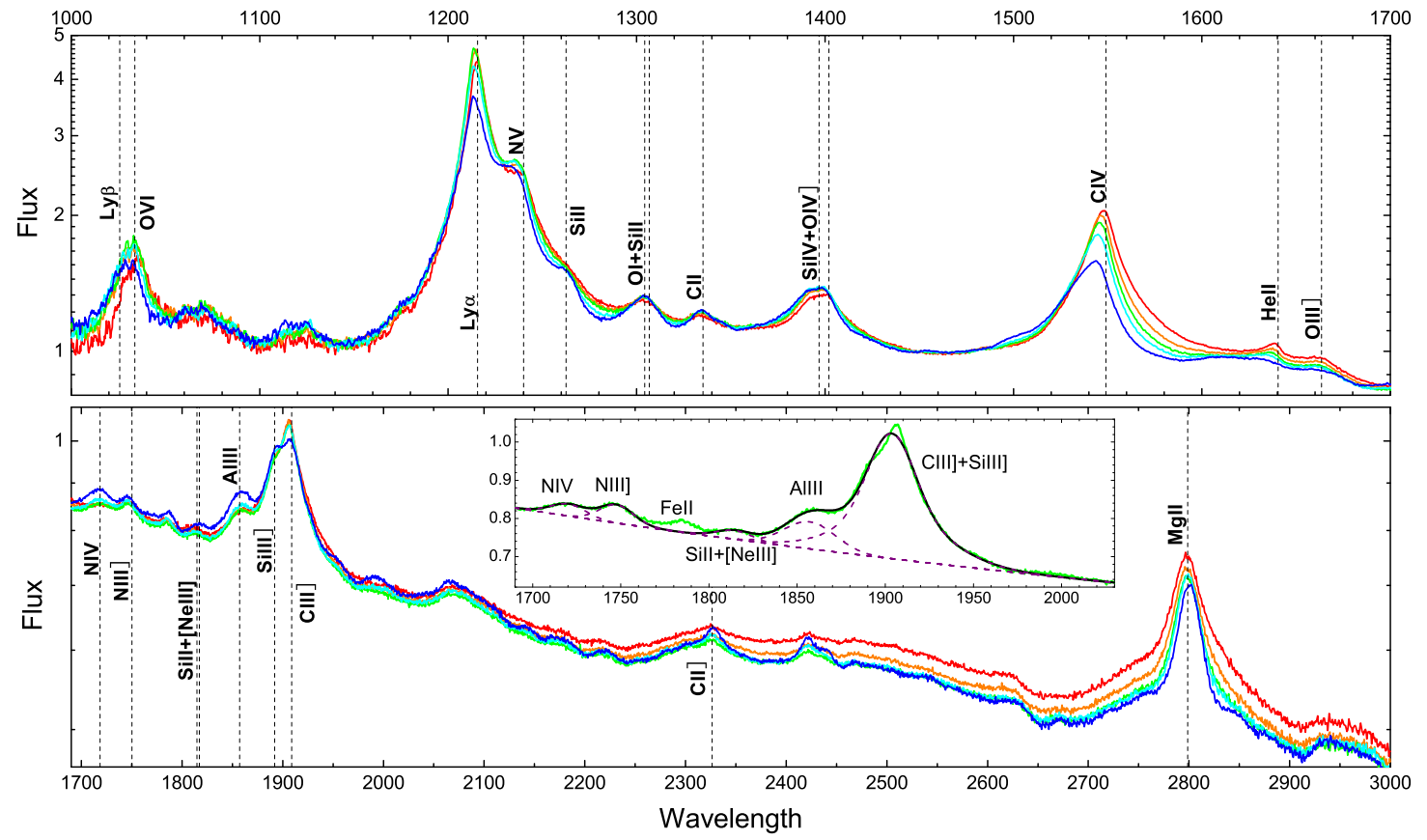

Fig. 1. - The composite quasar spectra, in the order of increasing BAI, are plotted as red, orange, green, cyan and blue, respectively. Most of the emission features are labelled. The dashed lines indicate the rest frame wavelength of the corresponding emission lines. The inset shows a demonstration of spectral fitting in $1700 \AA \sim 2300 \AA$ region for the middle-BAI composite (green line). The black line shows the best-fit model, the purple dashed lines show the fitting results for each emission line. The model is described in detail in Section 2.2 . 
Table 1: Continuum windows and number of Gaussians

\begin{tabular}{lccc}
\hline \hline Lines & $\lambda_{l o}(\AA)$ & $\lambda_{h i}(\AA)$ & Gaussians \\
\hline O VI+Ly $\beta$ & 1012 & 1055 & 1 \\
O I+Si II & 1286 & 1321 & 2 \\
C II & 1321 & 1353 & 2 \\
Si IV+O IV] & 1353 & 1450 & 3 \\
C IV & 1450 & 1695 & 3 \\
N IV & 1695 & 2030 & 1 \\
N III] & 1690 & 2030 & 1 \\
Si II+[Ne III] & 1690 & 2030 & 1 \\
Al III & 1690 & 2030 & 1 \\
C III]+Si III] & 1690 & 2030 & 2 \\
\hline
\end{tabular}

Note. - $\lambda_{l o}$ and $\lambda_{h i}$ indicate the center wavelengths of two continuum windows.

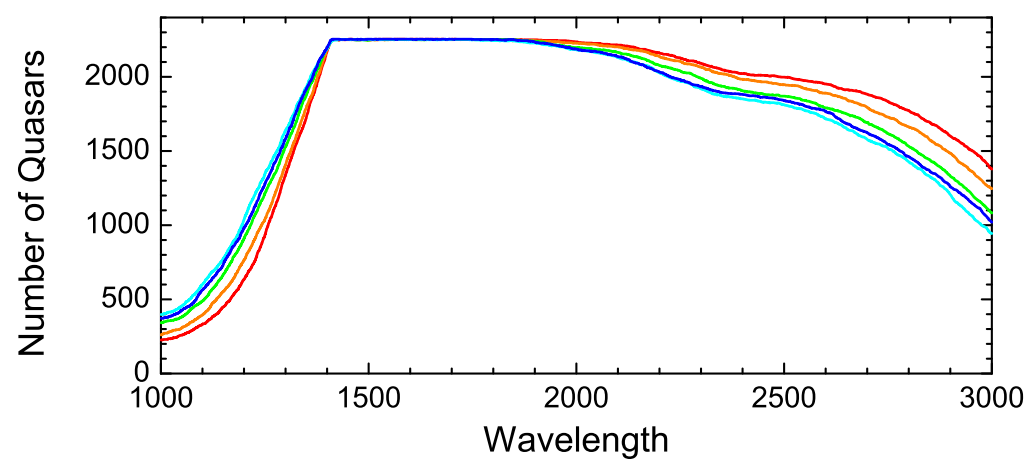

Fig. 2.- Number of quasars in each wavelength bin of the composites as a function of wavelength. The color code is the same as those in Figure 1. 


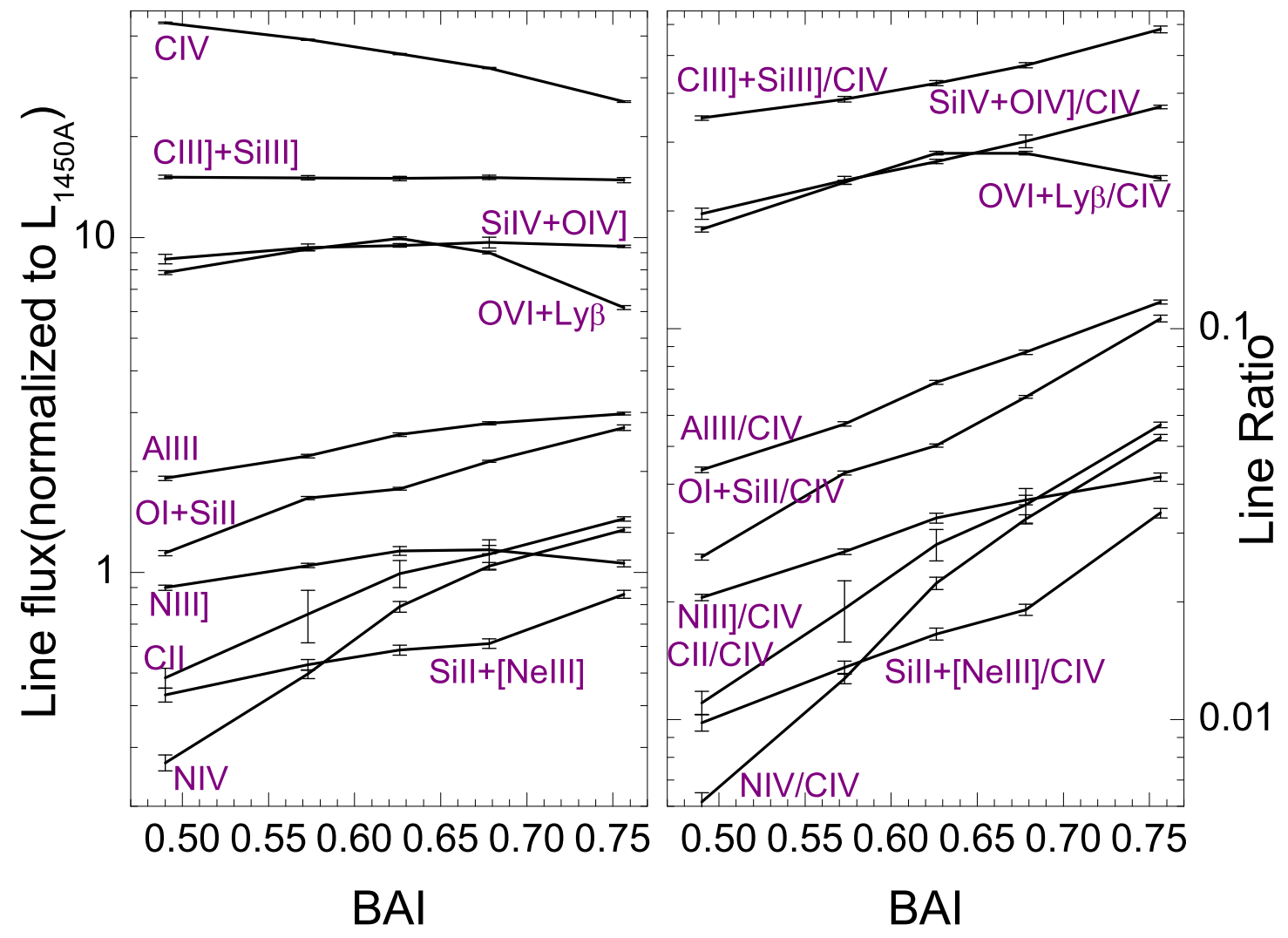

Fig. 3.- Left panel: Various line flux as a function of BAI. Right panels: The flux ratio of other lines to CIV as a function of BAI. All these quantities are measured from the composites. Note that $\mathrm{O}$ VI $+\mathrm{Ly} \beta$ are severely affected by Ly $\alpha$ forest. 


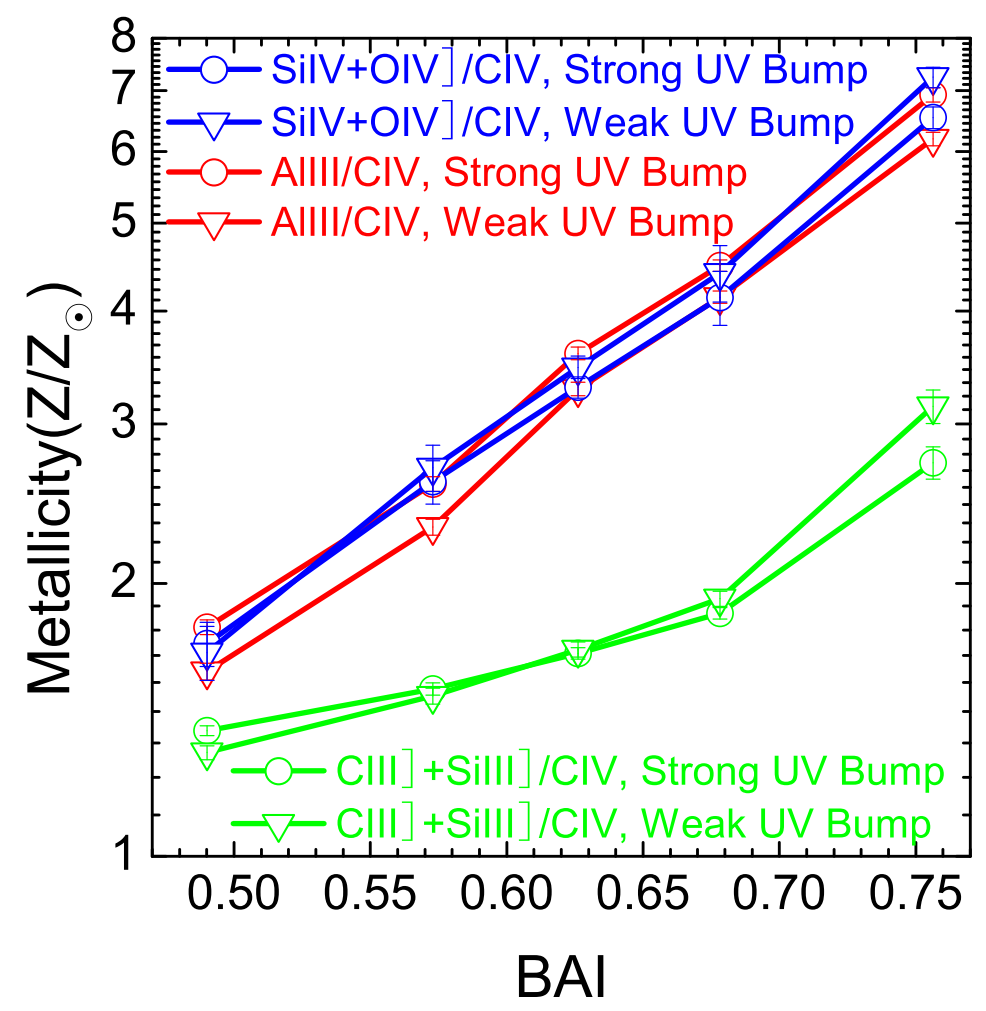

Fig. 4.- Metallicity inferred from different indicators (as indicated in the panel) as a function of BAI. Please see Nagao et al. (2006) for the details about the theoretical line ratio-metallicity relationships for different UV continuum models. 